\title{
Características y percepción de hogares con obesidad infantil en Cuenca, Ecuador
}

DOI: https://doi.org/10.21158/01208160.n88.2020.2694

Fecha de recepción: 17 de octubre de 2019

Fecha de aprobación: 19 de junio de 2020
Marco Antonio Ríos-Ponce ${ }^{1}$

Universidad del Azuay

mrios@uazuay.edu.ec

Nicole Doménica Guevara-Crespo ${ }^{2}$

Universidad del Azuay

niki1994@es.uazuay.edu.ec

Juan Francisco Álvarez-Valencia ${ }^{3}$

Universidad del Azuay

falvarezv@uazuay.edu.ec

Cómo citar este artículo / To reference this article / Comment citer cet article / Para citar este artigo:

Ríos-Ponce, M. A.; Guevara-Crespo, N. D.; Álvarez-Valencia, J. F. (2020). Características y percepción de hogares con obesidad infantil en Cuenca, Ecuador. Revista Escuela de Administración de Negocios, (88), 207-220. DOI: https://doi.org/10.21158/01208160. n88.2020.2694

\section{Resumen}

En Ecuador los índices de obesidad infantil presentan un aumento con respecto a 20 años atrás. Ante este panorama, el presente artículo tiene como objeto identificar las características de hogares con obesidad infantil en Cuenca, Ecuador, así como su percepción o comportamiento con respecto a herramientas de marketing tales como publicidad, promociones y merchandising en la obesidad infantil. Mediante una investigación de carácter exploratorio-descriptivo, transversal y cuantitativo, se identificó una correlación estadística entre las diferentes estrategias de marketing y la obesidad infantil, pues el $75 \%$ de los padres encuestados respondieron que las tácticas de promoción influyen en sus decisiones de compra. Fue posible determinar también que las anomalías en el peso de los niños — obesidad y sobrepeso — se ven condicionadas igualmente por factores externos, entre los cuales se encuentran elementos del marketing mix, como, por ejemplo, la publicidad en medios, así como en los puntos de venta, que influyen en el comportamiento de compra de los hogares y promueven así hábitos poco saludables para la población infantil. Se evidenció, por otra parte, que la obesidad incrementa en los niveles socioeconómicos más bajos. La actividad física se reemplaza por actividades de entretenimiento en medios; el $84 \%$ de los niños le dedica entre $1 \mathrm{~h} /$ día a $5 \mathrm{~h} /$ día. Finalmente, se destaca que el ejemplo de los padres o cuidadores es determinante en la problemática.

Palabras clave: marketing; marketing mix; merchandising; packaging; publicidad; comportamiento de compra; obesidad infantil.

\footnotetext{
${ }^{1}$ Ingeniero Comercial, Universidad del Azuay - Ecuador. Especialista en Administración de Empresas - Universidad de Buenos Aires Argentina. Posgrado en Marketing de Industria Farmacéutica - Universidad de Belgrano - Argentina. Master Business Administration - Universidad de Palermo - Argentina. ORCID: https://orcid.org/0000-0003-0586-680X

${ }^{2}$ Ingeniera en Marketing - Universidad del Azuay. Máster en Neuromarketing e Investigación de Mercados - Universitat de Barcelona. ORCID: https://orcid.org/0000-0003-1007-0929

${ }^{3}$ Ingeniero Comercial, Universidad del Azuay - Ecuador. Master Business Administration - Universidad de Belgrano - Argentina. ORCID: https://orcid.org/0000-0003-2876-4953
} 


\title{
Characteristics and perception of households with child obesity in Cuenca, Ecuador
}

\begin{abstract}
The rates of child obesity in Ecuador have increased over the previous 20 years. Given this panorama, this article aims at identifying the characteristics of households with child obesity in Cuenca, Ecuador, as well as their perception or behavior regarding child obesity and marketing tools such as advertising, promotions, and merchandising. It is by means of an exploratory-descriptive, cross-sectional, and quantitative research, that is possible to identify a statistical correlation between different marketing strategies and childhood obesity, as $75 \%$ of the parents that were surveyed responded that promotional tactics influence their purchasing decisions. It was also possible to determine that anomalies in children's weight - obesity and overweight - are conditioned by external factors, including elements from the marketing mix, such as advertising, not only in the media but at the point of sale, influencing household purchasing behavior and thus promoting unhealthy habits for the child population. On the other hand, it became clear that obesity increases in the lower socioeconomic levels. Physical activity is replaced by media entertainment activities: $84 \%$ of children spend between $1 \mathrm{~h} /$ day and $5 \mathrm{~h} /$ day. Finally, it should be noted that the example set by parents or caregivers is a determining factor in the problem.
\end{abstract}

Keywords: marketing; marketing mix; merchandising; packaging; advertising; purchasing behavior; child obesity.

\section{Características e percepção de lares com obesidade infantil em Cuenca, Equador}

\section{Resumo}

No Equador, as taxas de obesidade infantil mostram um crescimento em relação a 20 anos atrás. Diante desse contexto, este artigo tem como objetivo identificar as características dos lares com obesidade infantil em Cuenca, Equador, bem como sua percepção ou comportamento em relação às ferramentas de marketing como publicidade, divulgações e merchandising na obesidade infantil. Através de uma pesquisa exploratóriadescritiva, transversal e quantitativa, foi identificada uma correlação estatística entre as diferentes estratégias de marketing e de obesidade infantil, pois $75 \%$ dos pais entrevistados responderam que as táticas de divulgação influenciam em suas decisões de compra. Também foi possível constatar que as anomalias no peso das crianças - obesidade e sobrepeso - também são condicionadas por fatores externos, entre os quais estão elementos do Marketing mix, como, por exemplo, a publicidade nos meios de comunicação, bem como nos pontos de venda, que influenciam no comportamento de compra das famílias e, portanto, promovem hábitos não saudáveis para a população infantil. Por outro lado, evidenciou-se que a obesidade é maior nos níveis socioeconômicos mais baixos. A atividade física é substituída por atividades de entretenimento na mídia, $84 \%$ das crianças dedicam entre $1 \mathrm{~h} /$ dia a $5 \mathrm{~h} /$ dia. Finalmente, destaca-se que o exemplo dos pais ou cuidadores é decisivo no problema.

Palavras-chave: marketing; marketing mix; merchandising; embalagem; publicidade; comportamento de compra; obesidade infantil. 


\section{Résumé}

\section{Caractéristiques et comportement des foyers confrontés} à l'obésité infantile dans la ville de Cuenca en Équateur

En Équateur, les taux d'obésité infantile ont fortement progressé lors des vingt dernières annés. Cet article tente d'identifier les caractéristiques des foyers atteints par l'obésité infantile dans la ville de Cuenca, en Équateur, et d'analyser la perception et le comportement de cette population face au marketing, à la publicité, aux promotions et au merchandising. Grâce à une recherche exploratoire, descriptive, transversale et quantitative, il a été possible d'identifier une corrélation statistique entre les différentes stratégies marketing et l'obésité infantile: 75\% des parents interrogés ayant répondu que les tactiques promotionnelles influençaient leurs décisions d'achat. Nous avons d'une part pu déterminer que le surpoids des enfants - obésité - est également conditionné par des facteurs externes parmi lesquels des éléments du marketing mix, la publicité dans les médias ou les points de vente qui influencent le comportement d'achat des ménages et favorisent des habitudes de consommation malsaines chez la population enfantine. Il a été démontré par ailleurs que l’obésité augmente dans les couches socio-économiques les moins favorisées où l'activité physique est remplacée par des activités de divertissement médiatiques, $84 \%$ des enfants y consacrant entre une à cinq heures par jour. Enfin, il convient de souligner que l'exemple donné par les parents est déterminant dans la résolution de l’obésité infantile.

Mots-clés: marketing; mix marketing; marchandisage; emballage; publicité; comportement d'achat; obésité infantile. 


\section{Introducción}

$\mathrm{B}_{\perp}^{\mathrm{i}}$ iel (1990), mediante su investigación titulada Love the Ad. Buy the product?, comprobó que las acciones de marketing inciden en el comportamiento y las actitudes de los consumidores. Por tanto, ise puede considerar que elementos del marketing pueden influir en la percepción y el comportamiento de los sujetos, de modo que pueden ser un factor exógeno que incida en la obesidad infantil?

En la actualidad, la obesidad presenta un aumento de forma alarmante a nivel global, al punto que en los adultos es el principal problema de malnutrición y, cada vez más, en la población infantil (Chueca, Azcona y Oyarzábal, 2002). En el 2012, murieron en todo el mundo alrededor de 56 millones de personas, de las cuales 620000 murieron a causa de la violencia humana, 800000 se suicidaron y 1,5 millones como consecuencia de la diabetes (OMS, 2012). Noah (2016) asegura que «el azúcar es ahora más peligroso que la pólvora» (p. 30). Santos-Muñoz (2005) explica la obesidad como una enfermedad crónica de origen multifactorial, asociada a complicaciones de salud potencialmente graves; de acuerdo con estudios, el consumo de azúcar se relaciona con elementos sociales, fisiológicos, metabólicos, moleculares y genéticos.

Bajo la misma línea, Burrows (2000) se refiere a los coadyuvantes de la obesidad infantil:

la oferta excedida de productos elaborados hipercalóricos, un mayor poder adquisitivo, un marketing que induce al consumo, trastornos psicológicos reactivos donde el alimento pasa a ser un sustituto placentero y el desconocimiento, por parte de la población, que la obesidad es una enfermedad asociada a pequeños «desbalances» mantenidos en el tiempo. (p. 106)
Este artículo presenta un análisis cuantitativo y se concibe con el objetivo de analizar la percepción de hogares con niños de cinco a 11 años con obesidad, con respecto a distintas herramientas de marketing tales como publicidad en medios, promociones y merchandising en la obesidad infantil. Así mismo, identifica las principales características sociodemográficas de estos hogares en Cuenca, Ecuador.

La investigación reveló que la obesidad incrementa en aquellos niveles socioeconómicos (NSE) más bajos, de modo que existe una relación inversa entre estas variables $-\mathrm{p}=0,08$ - Además, se evidenció una relación entre el sexo del niño y la posibilidad de padecer algún grado de sobrepeso $u$ obesidad $-p=0,41-$.

Los escolares provienen de hogares con sociedades conyugales $-68 \%$ - Se determinó que el estado civil de los padres es un factor participante de la obesidad infantil $-\mathrm{p}<0,05-$. Sus progenitores tienen niveles de educación superior — universidad, pregrado y posgrado-. Las generaciones a las que pertenecen los padres, según la edad, son: baby boomers -26\%-, generación $\mathrm{X}-27 \%-\mathrm{y}$ millennials - $46 \%$ -

La actividad física se reemplaza por otras actividades de ocio, en gran parte por los medios electrónicos y las redes sociales, a los cuales los niños tienen acceso. Así, a diario, el 84,4\% de los niños dedica entre $1 \mathrm{~h}$ a $5 \mathrm{~h}$ a estos medios, además, se resalta el hábito de los hogares - 36,4 \% - de mirar televisión durante la ingesta de alimentos. Esto posibilita el desarrollo de obesidad y sobrepeso en los niños $-\mathrm{p}=0,01-$. Por otra parte, el $75,3 \%$ de los padres respondieron que tácticas de promoción tales como combos y descuentos influyen en sus decisiones de compra; se encontró esta variable como un factor que incide en el padecimiento de SP y OI $-\mathrm{p}=0,007-$. 


\section{Revisión de literatura}

$\mathrm{L}$ a obesidad es una enfermedad crónica producto de un desequilibrio en el consumo de alimentos, hábitos alimentarios poco saludables, el sedentarismo y factores psicosociales (Maldonado, 2018). Se da, en gran parte, en etapas en las que el desarrollo del ser humano atraviesa notables cambios tanto en su anatomía como en su psique; estas son durante la gestación, la lactancia, entre los cinco a siete años y en la adolescencia.

En relación con el sedentarismo, varios estudios han analizado aspectos tales como horas dedicadas al ocio, intensidad de actividad física y ejercicio realizado, entre otros. Como resultado se afirma que constituye uno de los factores de riesgo más importantes de la obesidad (Cambizaca, Castañeda, Sanabria y Morocho, 2016; Cigarroa, Sarqui y Zapata, 2016; González, 2010; Martínez, Moreno y Marques, 2002).

En Ecuador, tres de cada diez niños sufren de sobrepeso y obesidad, niños que se encuentran en edad escolar (Unicef, 28 de agosto de 2014). Según datos de la Encuesta Nacional de Salud y Nutrición (Freire et al., 2013), Ecuador registra un 8,6 \% de niños menores de cinco años con exceso de peso, mientras que en el rango de edad de cinco a 11 años el índice de obesidad se triplica y llega al 29,9\%. La Organización Mundial de la Salud (OMS) afirma que la publicidad y otras formas del marketing de alimentos y bebidas dirigidas a niños promueven, sobre todo, productos ricos en grasas o sal, y en este sentido expone que los medios utilizados por las organizaciones para comunicar y promocionar sus productos no nutritivos influyen en la obesidad infantil y en adolescentes, en razón a la facilidad al acceso que tienen los menores hoy en día a estos (OMS, 2010).
En la actualidad, las horas de inactividad física y sueño ocupan un porcentaje importante del día de muchos niños y adolescentes, especialmente en las zonas más urbanas (Burrows, 2000). De acuerdo con los investigadores De Onis y Blössner (2000), existe un patrón encontrado en los distintos estudios realizados en Latinoamérica en las altas tasas de obesidad en mujeres de bajos ingresos y el aumento de la obesidad en niños menores de cinco años de las ciudades más pobres.

El estudio de la Secretaría de Salud del Ecuador, realizado en el 2016, reveló que el $47 \%$ de 4000 muestras de alimentos - mercados municipales, bares escolares, comerciantes formales e informalestenían algún grado de contaminación y no cumplía la norma de calidad de alimentos (El Comercio, 2014). Bajo este marco, Pedraza (2009) describe una relación entre la condición socioeconómica y la obesidad que puede estar influenciada por el estadio de desarrollo social y económico de los diferentes países. En cuanto al estilo de vida de los niños, Tremblay y Willms (2003), por medio de un estudio con niños canadienses, explicaron cómo los hábitos que estos practiquen pueden generar riesgos asociados con el desarrollo de la obesidad. En este sentido, se identificaron las relaciones entre inactividad física y obesidad. Por su parte, Gortmaker et al. (1996) evidenciaron el exceso de mirar televisión como uno de los hábitos sedentarios más comunes de la niñez actual, y señalan esta en Norteamérica entre unas de las principales causas para la OI. 


\section{Metodología}

$\mathrm{E}^{\mathrm{n}}$ este artículo se presenta una investigación de carácter exploratoria-descriptiva transversal cuantitativa. Una investigación exploratoria consiste en:

examinar una determinada situación para ampliar el objetivo de estudio, pudiendo utilizarse para definir un problema con mayor precisión o establecer hipótesis, descubrir las variables y relaciones más relevantes y establecer prioridades para poder avanzar en la investigación posterior o futura. Se considera descriptiva ya que el objetivo es especificar una situación del mercado o las características de los consumidores, de naturaleza transversal debido a que es realizada en un único momento en el tiempo con una muestra determinada. (Esteban y Molina, 2014)

\subsection{Objetivos}

\subsubsection{Objetivo general.}

El objetivo general de la investigación fue identificar las características de los hogares con obesidad infantil de Cuenca, Ecuador, así como su percepción o comportamiento con respecto a herramientas de marketing tales como publicidad, promociones $y$ merchandising en la obesidad infantil.

\subsubsection{Objetivos específicos.}

Los objetivos específicos fueron dos:

- identificar la percepción de hogares con obesidad infantil en relación con elementos del marketing;

- recopilar datos que permitan identificar características sociodemográficas de los hogares con obesidad infantil.

\subsection{Diseño muestral}

En primera instancia, se obtuvo la autorización de las escuelas para la realización del estudio y se entregaron hojas de consentimiento a los padres para colaborar en la investigación; además, se expresó que el tratamiento estadístico de la información aportada sería anónimo.

El tamaño mínimo de muestra, para la población urbana de Cuenca de 277374 habitantes, bajo un nivel de confianza del $95 \%$ y considerando un error relativo del $5 \%$ se estimó en 384 hogares.

Se realizó un muestreo estratificado aleatorio simple, se estudiaron 100 escuelas comprendidas entre fiscales, fiscomisionales y privadas; se recabó información de un total de 2555 individuos, 1286 niños — 50,3 \% - y 1269 niñas — 49,7 \%—, de los cuales presentaron sobrepeso y obesidad un total de 396. El periodo del estudio fue desde septiembre del 2018 hasta diciembre del 2018.

\subsection{Instrumento}

La recopilación de la información se realizó por medio de un cuestionario previamente construido por los autores. Posterior a su aplicación se aplicó un análisis estadístico — frecuencias y chi cuadradopor medio de tratamiento estadístico. 


\section{Análisis de los resultados}

Qus progenitores tienen niveles de educación superior — universidad, pregrado y posgrado-. Las generaciones a las que pertenecen los padres, según la edad, son: baby boomers -26\%-, generación $\mathrm{X}-27 \%$ - y millennials - $46 \%$ -

El nivel de instrucción de los representantes de los niños resultó ser un factor influyente para el desarrollo de obesidad $-\mathrm{p}=0,00-$. Aquellos hogares con padres con un nivel superior - universidad- de educación presentaron casos de mayor sobrepeso infantil (véase la figura 1).
El estudio de Márquez-Díaz (2016) analizó que existe una gradiente inversa en función de los estudios:

mientras que el 26,7 \% de la población adulta con educación primaria o inferior era obesa, ese porcentaje fue del 19,0 \% y del 13,3\% en los sujetos con educación secundaria de primer y segundo ciclo respectivamente, y del 9,8\% en los de estudios universitarios. (p. 185)

Figura 1. Tasa de sobrepeso y obesidad infantil en hogares con padres con estudios de tercer nivel

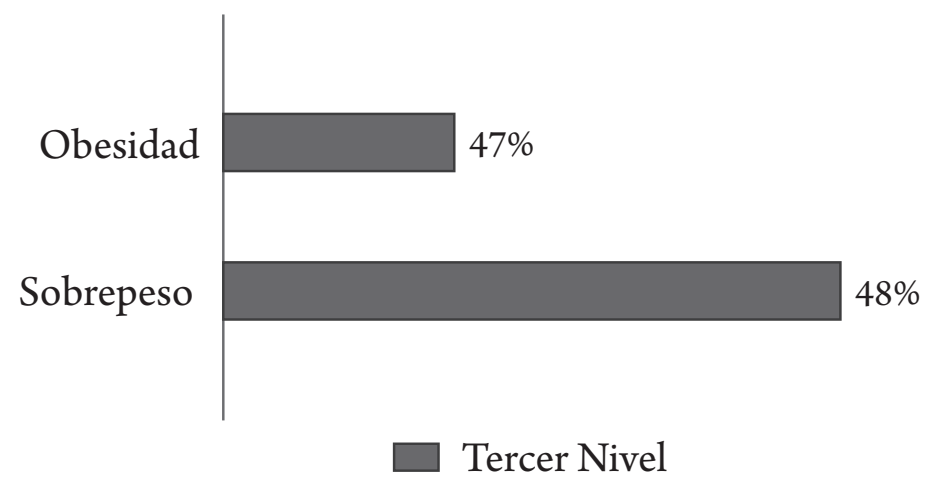

Fuente. Elaboración propia.

En relación con la variable de estado civil, los niños que pertenecían a un núcleo familiar de padre y madre presentaron mayores índices de obesidad (véase la figura 2). Por otra parte, el $33 \%$ de los niños que tienen obesidad son procedentes de padres divorciados. Se determinó que el estado civil de los representantes del niño contribuye a que este padezca de obesidad o SP $-p=0,05-$. 
Figura 2. Sobrepeso y obesidad en niños de padres casados

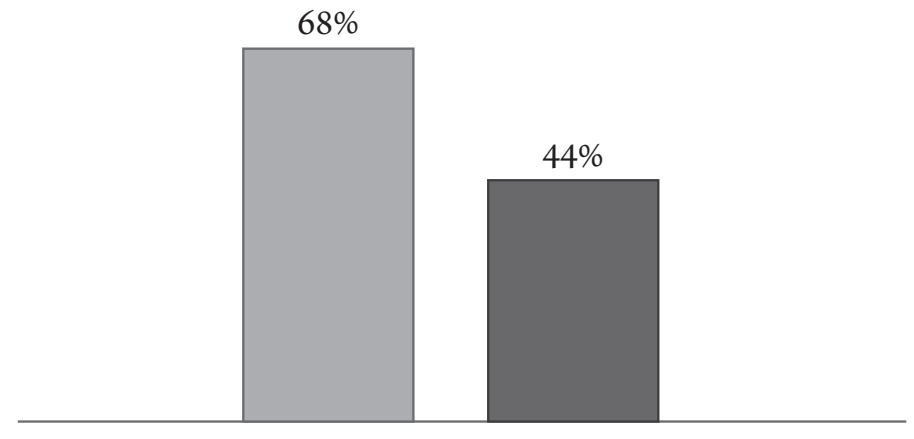

Representantes casados

Sobrepeso $\square$ Obesidad

Fuente. Elaboración propia.

La actividad física se reemplaza por otras actividades de ocio, en gran parte por los medios electrónicos ylas redes sociales a los que los niños tienen acceso (véase la figura 3). El 84,4 \% de los niños dedica tiempo a los medios, entre $1 \mathrm{~h}$ a $5 \mathrm{~h}$ diarias. Es importante notar que, de acuerdo con investigaciones, el uso de un medio no excluye el uso de otro. Es decir, un niño puede mirar televisión y también interactuar con una computadora. De manera general, el tiempo promedio al que son expuestos los niños a medios - televisión, computadora, celular, tablet - supera las 20 horas semanales, y como consecuencia se desplaza la realización de actividades físicas moderadas y vigorosas.
Bajo ese contexto, se han realizado estudios desde la década de los ochenta, los cuales revelan que es factible disminuir el peso corporal de los niños reduciendo el tiempo que pasan con los medios (Kaiser Family Foundation, 2003; Kaiser Family Foundation, 2005; Reilly, Dorosty y Emmet, 1999; Rideout, 2004). En el Reino Unido se analizó el contenido comunicacional de la publicidad alimentaria dirigida a menores en el 2008, y se evidenció que el 71,2\% de los comerciales de alimentos y bebidas, emitidos durante la franja horaria de protección reforzada, anunciaban productos con características nutricionales pobres (Vásquez et al., 2013). Por su parte, el estudio de Moreno y Toro (2009) reveló que el 41 \% de los entrevistados pasan entre una a tres horas frente a la televisión, mientras el $29 \%$ más de tres horas.

Figura 3. Tasa de acceso de los niños a los medios

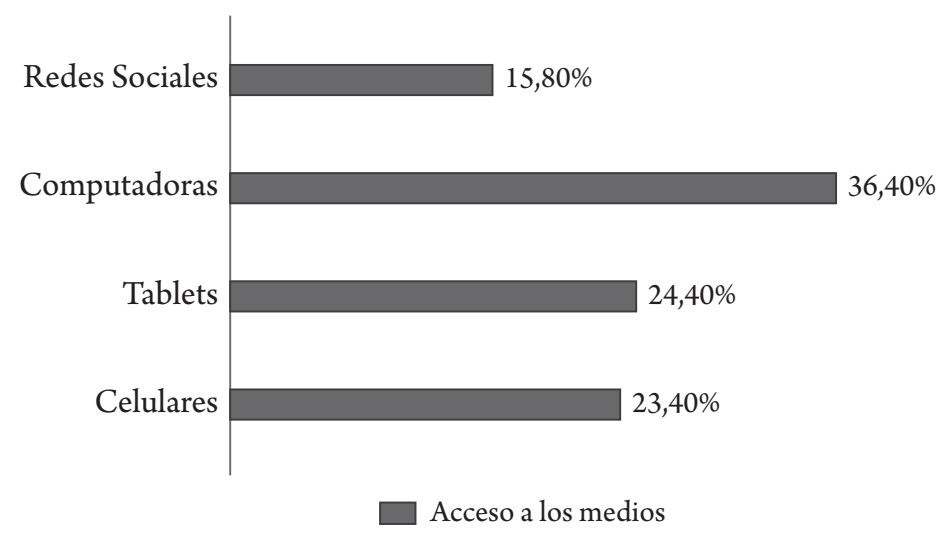

Fuente. Elaboración propia. 
Como consecuencia, el $59 \%$ de los niños no realiza ningún tipo de actividad física. La OMS cataloga la disminución de actividad física en niños y adolescentes como un factor atribuible al padecimiento de obesidad (OMS, 4 de marzo de 2018). La ausencia de ejercicio en la niñez, cuando no se padece obesidad, señala no ser atribuible $-\mathrm{p}=$ 0,22—; por el contrario, si el niño es obeso o indica tener probabilidades de serlo, el ejercicio físico toma un rol determinante $-\mathrm{p}=0,04-$.

Figura 4. Habitación común para la TV en casa
De los medios mencionados, con los cuales los niños interactúan, la televisión es aquel al cual más están expuestos: el 89,5\% de los hogares tienen en casa entre uno a tres televisores, y la tasa de acceso de los niños a este medio es del 62,5\%. Se identificó que la moda para ubicar este objeto es hacerlo en el dormitorio de los padres y en la sala de estar (véase la figura 4).

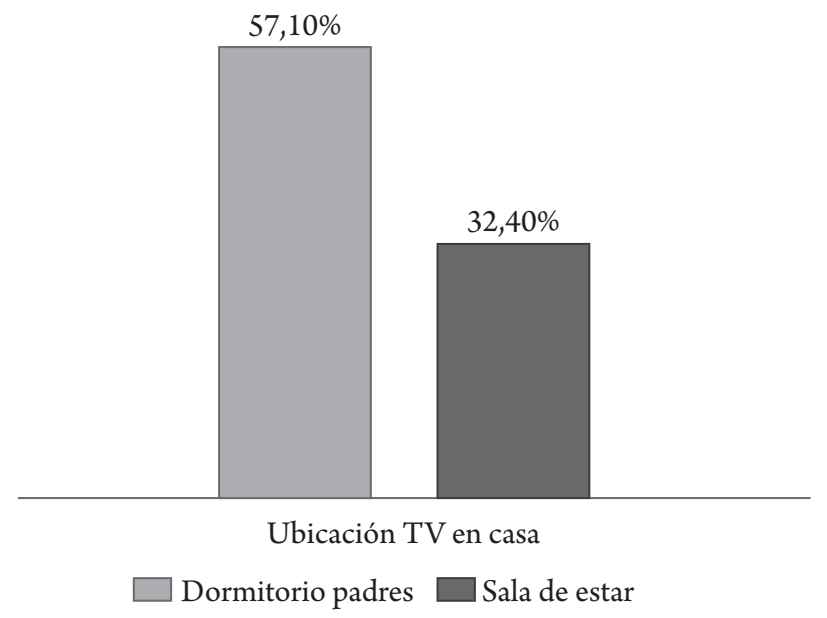

Fuente. Elaboración propia.

En cuanto al estilo de vida de los niños, Tremblay y Willms (2003), por medio de un estudio con niños canadienses, explicaron cómo los hábitos que estos practiquen pueden generar riesgos asociados con el desarrollo de la obesidad. En este sentido, en la presente investigación se identificaron las relaciones entre inactividad física y obesidad. Cabe mencionar que se manifestó una tendencia hacia el tiempo y la comodidad como factores de mayor peso en sus decisiones de alimentación y actividad física.

Además, se resalta el hábito de los hogares — 36,4\%de mirar televisión durante la ingesta de alimentos, lo que posibilita el desarrollo de sobrepeso u obesidad en los niños $-\mathrm{p}=0,01-$. En general, la exposición a medios electrónicos favorece a la problemática $-\mathrm{p}$ $=0,02-$. Se ha evidenciado a través de estudios que la televisión es uno de los hábitos sedentarios más comunes de la niñez actual, y cómo es unas de las principales causas para la obesidad infantil (Borges et al., 2007; Gortmaker et al., 1996; Martínez et al., 2011; Moreno y Toro, 2009).

Los ingresos familiares oscilan entre los USD 386 y USD 772 — $37 \%$ - y entre los USD 773 y USD 1158 - $40 \%$-, con una media de USD 772,25. La investigación reveló que la obesidad incrementa en aquellos niveles socioeconómicos (NSE) más bajos, de modo que existe una relación inversa entre estas variables $-\mathrm{p}=0,08-$. Además, se evidenció una relación entre el sexo del niño y la posibilidad de padecer algún grado de sobrepeso $\mathrm{u}$ obesidad $-\mathrm{p}=$ 0,41 - que recae sobre el sexo femenino.

De acuerdo con De Onis y Blössner (2000), existe un patrón en los distintos estudios llevados a cabo en Latinoamérica: las altas tasas de obesidad en mujeres de bajos ingresos y el aumento de la obesidad en niños 
menores de cinco años de las ciudades más pobres. En Brasil, por ejemplo, el Gobierno realizó un estudio en 1989 que indicaba un aumento en las prevalencias de la obesidad, según el cual 6,8 millones $-8 \%$ de individuos adultos presentaron obesidad y cerca de 27 millones - $32 \%$ - algún grado de sobrepeso; de estos resultados el predominio vino dado por el sexo femenino, con el $38 \%$ de la población y el $27 \%$ masculino (Ferreria y Magalhães, 2006).
Por otra parte, el sitio donde se preparan los alimentos es un determinante para el sobrepeso $u$ obesidad $-\mathrm{p}=0,00$ - Preparar los alimentos en casa es una costumbre muy arraigada en la cultura ecuatorianacuencana —el 91,5\% de los hogares lo afirman-. Sin embargo, esto no indica que la comida hogareña sea más saludable (véase la figura 5). Así mismo, en los hogares de menores recursos económicos la compra de comestibles se realiza en mercados tradicionales. En los hogares con mayores posibilidades se realiza en lugares que brindan comodidad.

Figura 5. Niños con sobrepeso y obesidad que se alimentan en casa

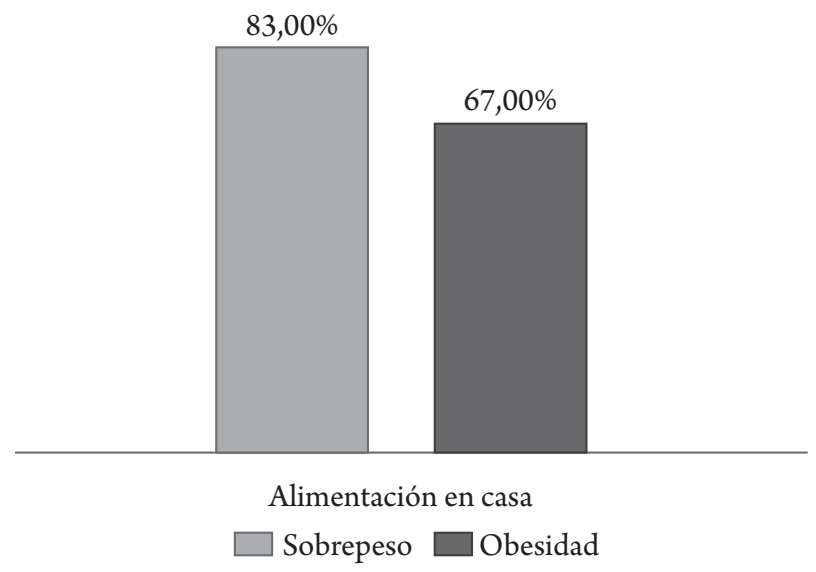

Fuente. Elaboración autores.

Este resultado se relaciona con la falta de conocimiento acerca del contenido de los productos que consume y con los cuales alimenta a su familia. El 72,7 \% señala consumir productos orgánicos, pero el estudio reveló que su consumo para este escenario contribuye al SP y $\mathrm{OI}-\mathrm{p}=0,011-$ Al profundizar en este resultado, se encontraron dos factores claves: el poco interés de la población por verificar el contenido, las propiedades y los beneficios reales de los productos alimentarios apenas el $34 \%$ lo hace siempre-y la publicidad que se realiza para estos productos.

Con relación al marketing, la percepción de los hogares reveló que los envases con sponsor ejercen influencia en el deseo de compra de sus hijos, lo cual evidencia el packaging como un factor motivante para el sobrepeso u obesidad — p = 0,00—. El $95 \%$ de los padres notó que sus hijos se fijan más en las marcas que en el contenido del producto, sin embargo, esto no indica ser un factor coadyuvante para esta enfermedad. Así mismo, el 76,6 \% de los padres manifestó que las técnicas de merchandising —exhibición de góndolas y puntos de venta - influyen en sus decisiones de compra y los deseos de compra de sus hijos. El estudio indicó que el merchandising es una herramienta de marketing efectiva para influir en los individuos y como producto aporta al desarrollo de SP y OI $-p$ $=0,01-$. En la sociedad en estudio es muy común encontrar promociones y combos, los cuales atraen, la mayoría de veces, gran afluencia de gente. El 75,3\% de los padres respondieron que estas tácticas de marketing influyen en su decisión de compra, y, por tanto, pueden incidir en el padecimiento de sobrepeso u obesidad $-\mathrm{p}=0,00-$. 
En su mayoría, los padres/adultos responsables no cocinan, acuden a una tercera persona que realiza los almuerzos. Se destacan tres actores encargados de cocinar los alimentos: la madre — 49\%—, los abuelos - $17 \%$ - y los viandistas $-12 \%$ - Los hogares prefieren, a la hora del almuerzo, consumir solo el plato fuerte -61\%—, el cual está caracterizado por una porción notable de carbohidratos, entre los cuales los más citados son el arroz, los fideos y las papas.

El consumo de golosinas y snacks a la semana tiende a ser habitual, oscilando entre 20 y 25 unidades a la semana. El consumo de golosinas es habitual en los escolares: cerca del $31 \%$ las consume con mucha frecuencia y el $16 \%$ todos los días. Se halló que el $30 \%$ de los niños consume, al menos una vez al día, algún tipo de bebida azucarada y carbonatada. El consumo de alimentos y bebidas azucaradas presenta una correlación positiva con la aparición de sobrepeso y obesidad en preescolares y niños mayores (Cambizaca et al., 2016; Dubois, Farmer, Girard y Peterson, 2007; Oliva y Fragoso, 2013; Popkin, 2004). Banwell, Lim, Sleigh, Bain, Dixon y Sleigh (2009) indicaron que, si en el mundo a nivel poblacional se eliminara el sedentarismo y el consumo de alimentos hipercalóricos y saturados en grasas, se disminuiría en un $31 \%$ la obesidad.

Por el contrario, la ingesta de verduras y frutas es baja. Los adultos manifestaron no gustar de estos alimentos, lo que explica por qué los niños no las consumen. La tasa diaria de consumo de frutas y verduras en los niños apenas alcanza el $17 \%$ y el $11 \%$, respectivamente. El consumo de lácteos al día, puntualmente leche, es del $34 \%$, leche entera — $45 \%$ - y semidescremada $-31 \%$ - Ante esto,
Birch y Ventura (2009) señalan que las prácticas alimentarias de los padres tienen un rol crucial, como, por ejemplo, exponer temprano a los niños a ciertos tipos de alimentos o enseñarles a que la alimentación está ligada a aspectos de disponibilidad de comida, aspectos que posibilitan la aparición y la prevalencia de la enfermedad en los menores.

Se indagó acerca del conocimiento que los progenitores tienen sobre la obesidad infantil. A pesar de que una gran mayoría de adultos expresó conocer sobre la obesidad $-76 \%$ e indicaron que está relacionada con el sedentarismo - 53\%- y una alimentación deficiente - $47 \%$-, sus hábitos se mostraron vinculados con las causas mencionadas. En el estudio de Ruvalcaba et al. (2018), el $100 \%$ de las personas poseía conocimiento del concepto de obesidad infantil, sin embargo, mantenían hábitos tales como el sedentarismo y el consumo de comida chatarra. Los autores concluyeron que «el problema no nace en el conocimiento de los factores causales, sino en los factores culturales y carencias de sitios para la adecuada nutrición» (p. 620).

Uno de los objetos resultantes de la investigación es la generación de una propuesta de campaña de marketing social para la temática abordada. Se procedió a consultar a los progenitores sobre el tipo de comunicación que más los impactaría y los motivaría a un cambio de comportamiento. Una campaña directa $-47 \%$ - fue la respuesta predominante, es decir, un tipo de diseño comunicacional que cale las emociones y los sentimientos de los receptores. Los medios predilectos para la comunicación fueron las redes sociales $-32 \%$ - y la televisión $-28 \%$ - 


\section{Conclusiones}

n conclusión, con respecto a las variables
analizadas se destaca lo siguiente.

Las anomalías en el peso de los niños —obesidad y sobrepeso- se ven condicionadas también por factores externos, entre los cuales se encuentran elementos del marketing mix, como, por ejemplo, la publicidad en medios, así como en los puntos de venta, que influyen en el comportamiento de compra de los hogares y promueven así hábitos poco saludables para la población infantil. Finalmente, el estudio permitió identificar las características de los hogares de Cuenca, Ecuador, con niños con obesidad y sin obesidad (véase la tabla 1 ).

Tabla 1. Paralelo de perfiles de hogares con niños obesos y niños con normopeso

\begin{tabular}{|c|c|}
\hline Niños con obesidad infantil & Niños sin obesidad infantil \\
\hline $\begin{array}{l}\text { Se caracterizan por ser hogares con casa propia } \\
-49 \% \text {-. }\end{array}$ & $\begin{array}{l}\text { Se caracterizan por ser hogares con casa propia } \\
-42 \% \text {. }\end{array}$ \\
\hline $\begin{array}{l}\text { La edad de los padres oscila entre los } 28 \text { a los } 37 \text { años } \\
-59 \% \text { - }\end{array}$ & $\begin{array}{l}\text { La edad de los padres oscila entre los } 28 \text { a } 37 \text { años } \\
-54 \% \text { - }\end{array}$ \\
\hline $\begin{array}{l}\text { La preparación de los alimentos acostumbran a hacerla } \\
\text { en casa }-82 \% \text { - }\end{array}$ & $\begin{array}{l}\text { La preparación de los alimentos acostumbran a hacerla } \\
\text { en casa }-92 \% \text { - }\end{array}$ \\
\hline $\begin{array}{l}\text { Los padres tienen un nivel de educación de secundaria } \\
-28 \%-\text { y tercer nivel }-49 \% \text {-. }\end{array}$ & $\begin{array}{l}\text { Los padres tienen un nivel de educación de secundaria } \\
-36 \% \text { - y tercer nivel }-42 \% \text {-. }\end{array}$ \\
\hline $\begin{array}{l}\text { El acceso a medios es un elemento que incide en el } \\
\text { desarrollo de la OI }-p=0,009-\text {. }\end{array}$ & $\begin{array}{l}\text { No hay relación entre su peso y el acceso a redes sociales } \\
-p=0,446-\end{array}$ \\
\hline $\begin{array}{l}\text { El nivel socioeconómico de los hogares es un factor } \\
\text { participante para el desarrollo de la OI }-p=0,000-\text {. }\end{array}$ & $\begin{array}{l}\text { El nivel socioeconómico de los hogares es un factor que } \\
\text { puede incidir en el peso de los niños }-p=0,013-\text {. }\end{array}$ \\
\hline $\begin{array}{l}\text { El } 61 \% \text { de los niños tienen acceso a varios medios - } \\
\text { TV, computadora, celular, redes sociales-. }\end{array}$ & $\begin{array}{l}\text { El acceso a medios como TV, computadora, celular y } \\
\text { redes sociales en los niños es de } 55 \% \text {. }\end{array}$ \\
\hline $\begin{array}{l}\text { Los niños dedican más de tres horas/día a medios } \\
\text { electrónicos - } 56 \% \text {-. }\end{array}$ & $\begin{array}{l}\text { El tiempo al día dedicado a medios electrónicos oscila } \\
\text { entre dos horas o menos }-63 \% \text {-. }\end{array}$ \\
\hline El $78 \%$ de los hogares disponen de internet. & El $76 \%$ de los hogares disponen de internet. \\
\hline $\begin{array}{l}\text { Existe el hábito de mirar televisión a la hora de comer. } \\
\text { En el caso de niños con SP y OI este comportamiento } \\
\text { coadyuva a su enfermedad }-\mathrm{p}=0,019-\end{array}$ & $\begin{array}{l}\text { Mirar televisión a la hora de comer en niños con un peso } \\
\text { bajo o normal no constituye un factor que influya en su } \\
\text { peso - } \mathrm{p}=0,890-\text {. }\end{array}$ \\
\hline $\begin{array}{l}\text { Los padres gustan recompensar a sus hijos con entrete- } \\
\text { nimiento }-33 \%-\text { y golosinas }-28 \%-\text {. }\end{array}$ & $\begin{array}{l}\text { Las recompensas a sus hijos en su mayoría son juguetes } \\
-38 \%-\text {, frutas }-22 \%-\text { y golosinas }-12 \%-\text {. }\end{array}$ \\
\hline $\begin{array}{l}\text { El tipo de recompensas o « gustos» que los padres den } \\
\text { a sus hijos es un elemento influyente en su peso }-p= \\
0,018) \text {. }\end{array}$ & $\begin{array}{l}\text { El tipo de recompensas o «gustos» que los padres den } \\
\text { a sus hijos es un elemento influyente en su peso }-p= \\
0,00-\text {. }\end{array}$ \\
\hline
\end{tabular}

Fuente. Elaboración propia. 


\section{Referencias}

Banwell, C.; Lim, L.; Seubsman, S.; Bain, C.; Dixon, J.; Sleigh, A. (2009). Body mass index and health-related behaviours in a national cohort of 87,134 Thai open university students. Journal of Epidemiology and Community Health, 63(5), 366372. DOI: https://doi.org/10.1136/jech.2008.080820

Biel, A. (1990). Love the Ad. Buy the product? Recuperado de https://bit.ly/2HG5YWc

Birch, L.; Ventura, A. (2009). Preventing childhood obesity: what works? International Journal of Obesity, 33(Suppl 1), S78-S81. DOI: https://doi.org/10.1038/ijo.2009.22

Borges, C.; Kraft, M.; Lima, M.; Fernández, A.; Teramoto, A.; Kanunfre, C. (2007). Influencia da televisao na prevalencia de obesidade infantil em Ponta Grossa, Paraná. Ciencia Cuidado e Saude, 6(3), 305-311.

Burrows, R. (2000). Prevención y tratamiento de la obesidad desde la niñez: la estrategia para disminuir las enfermedades crónicas no transmisibles del adulto. Revista Médica de Chile, 128(1), 105-110. DOI: https://doi.org/10.4067/S003498872000000100015

Cambizaca, G., Castañeda, I.; Sanabria, G.; Morocho, L. (2016). Factores que predisponen al sobrepeso y obesidad en estudiantes de colegios fiscales del Cantón Loja-Ecuador. Revista Habanera de Ciencias Médicas, 15(2), 163-176.

Chueca, M.; Azcona, C.; Oyarzábal, M. (2002). Obesidad infantil. Anales del sistema sanitario de Navarra, 25, 127-141.

Cigarroa, I.; Sarqui, C.; Zapata, R. (2016). Efectos del sedentarismo y obesidad en el desarrollo psicomotor en niños y niñas: una revisión de la actualidad latinoamericana. Revista Universidad y Salud, 18(1), 156-169. DOI: https:// doi.org/10.22267/rus.161801.27

Dubois, L.; Farmer, A.; Girard, M.; Peterson, K. (2007). Preschool children's eating behaviours are related to dietary adequacy and body weight. European Journal of Clinical Nutrition, 61(7), 846-855. DOI: https://doi.org/10.1038/sj.ejcn.1602586

El Comercio. (26 de agosto de 2014). La obesidad es una epidemia que golpea a Ecuador con fuerza. Recuperado de https://bit. ly/3mZSm7R

Esteban, A.; Molina, A. (Coord.). (2014). Investigación de mercados. Madrid: ESIC.

Ferreria, V.; Magalhães, R. (2006). Obesidade no Brasil: tendências atuais. Revista Portuguesa de Saúde Pública, 71-81.

Freire, W.; Ramírez, M. J.; Belmont, P.; Mendieta, M.; Silva, K.; Romero, N.; Sáenz, K.; Piñeros, P.; Gómez, L. F.;Monge, R. (2013). Encuesta Nacional de Salud y Nutrición del Ecuador. Quito: Ministerio de Salud Pública, Instituto Nacional de Estadísticas y Censos. Recuperado de https://bit. ly/37KCyBn
González, E. (2010). Obesidad. Un enfoque multidisciplinario. Hidalgo, México: Universidad Autónoma del Estado de Hidalgo. Recuperado de https://bit.ly/35x1wRS

Gortmaker, S.; Must, A.; Sobol, A.; Patterson, K.; Colditz, G.; Diets, W. (1996). Television viewing as a cause of increasing obesity among children in the United States. Archives of Pediatrics and Adolescent Medicine, 150 (4), 356-362. DOI: https://doi.org/10.1001/archpedi.1996.02170290022003

Kaiser Family Foundation. (2003). Kaiser Family Foundation. (2003). Kaiser Family Foundation. de Understanding the HealthCare Needs and Experiences of People with Disabilities: Findings from a 2003 Survey. Recuperado de https://bit.ly/2I71LeM

Kaiser Family Foundation. (2005). The effects of electronic media on children ages zero-six: a history of research-issue brief. Recuperado de https://bit.ly/3jzHcVc

Maldonado, A. (2 de mayo de 2018). ¿Cuánto sabemos de la obesidad infantil en Ecuador? UTPL Sala de redacción. Recuperado de https://bit.ly/2JH9rVF

Márquez-Díaz, R. (2016). Obesidad: prevalencia y relación con el nivel educativo en España. Nutrición clínica y dietética hospitalaria 36(3), 181-188.

Martínez, J.; Moreno, M.; Marques, A. (2002). Causas de obesidad. Anales Sis San Navarra, 25(Supl. 1),17-27.

Martínez, M.; García, P.; Aguilar, R.; Vázquez, L.; Gutiérrez, G.; Cerda, R. (2011). Asociación sobrepeso-obesidad y tiempo de ver televisión en preescolares. Ciudad fronteriza Noreste de México. Revista Enfermería Universitaria, 8(2), 12-17.

Moreno, A.; Toro, L. (2009). La televisión, mediadora entre consumismo y obesidad. Revista Chilena de Nutrición, 36(1), 46-52. DOI: https://doi.org/10.4067/S071775182009000100005

Noah, Y. (2016). Homo Deus: Breve historia del mañana. Barcelona: Debate.

Oliva, O.; Fragoso, S. (2013). Consumo de comida rápida y obesidad, el poder de la buena alimentación en la salud. Revista Iberoamericana para la Investigación y el Desarrollo Educativo, 4(7), 176-199. DOI: https://doi.org/10.23913/ride.v4i7.93

OMS (Organización Mundial de la Salud). (2010). Conjunto de recomendaciones sobre la promoción de alimentos y bebidas no alcohólicas dirigida a los niños. Recuperado de https://bit. ly/3edmmKl

OMS (Organización Mundial de la Salud). (4 de marzo de 2018). Datos sobre la obesidad. Organización Mundial de la Salud. Recuperado de https://bit.ly/31PfIVp

OMS (Organización Mundial de la Salud). (2012). Disease burden and mortality estimates. Recuperado de https://bit. ly/3k5BryG 
Onis, M. de; Blössner, M. (2000). Prevalence and trends of overweight among preschool children in developing countries. American Journal of Clinical Nutrition, 72(2), 1032-1039. DOI: https://doi.org/10.1093/ajcn/72.4.1032

Pedraza, D. F. (2009). Obesidad y pobreza: marco conceptual para su análisis en Latinoamérica. Saúde e Sociedade, 18, 103-117. DOI: https://doi.org/10.1590/S0104-12902009000100011

Popkin, B. G. L. (2004). The nutrition transition: worldwide obesity. International Journal of Obesity, 28(Supple 3), S2-S9. DOI: https://doi.org/10.1038/sj.ijo.0802804

Reilly, J.; Dorosty, A.; Emmet, P. (1999). Prevalence of overweight and obesity in British children: cohort study. BMJ, 319, 1039. DOI: https://doi.org/10.1136/bmj.319.7216.1039

Rideout, V. (01 de febrero de 2004). The Role of Media in Childhood Obesity - Issue Brief. Recuperado de https://bit.ly/32h0BnM

Ruvalcaba, J.; Hernández, J.; García, J.; Lozano, A.; Morales, L.; Hernández Medina, S.; Isla, I.; Rivera, M.; Reynoso, J. (2018). Factores desencadenantes de obesidad infantil, un problema de salud pública. Journal of Negative \&' No Positive Results, 3(8), 614-626. DOI: https://doi.org/10.19230/jonnpr.2542
Santos-Muñoz, S. (2005). La educación física escolar ante el problema de la obesidad y el sobrepeso. Revista Internacional de Medicina y Ciencias de la Actividad Física y del Deporte, 5(9), 179-199.

Tremblay, M.; Willms, J. (2003). Is the Canadian childhood obesity epidemic related to physical inactivity? International Journal of Obesity, 27(9), 1100-1105. DOI: https://doi. org/10.1038/sj.ijo.0802376

Unicef. (28 de agosto de 2014). Obesidad infantil. Recuperado de https://www.unicef.org/ecuador/obesidad-infantil

Vásquez, F.; Díaz, E.; Lera, L.; Vásquez, L.; Anziani, A.; Leyton, B.; Burrows, R. (2013). Evaluación longitudinal de la composición corporal por diferentes métodos como producto de una intervención integral para tratar la obesidad en escolares chilenos. Nutrición Hospitalaria, 28(1), 148-154. 